\title{
Posmodernidad, calidad y cantidad del gasto en la carrera de estado educativo en el Ecuador: alcances de educación superior y educación intercultural ${ }^{*}$
}

Posmodernity, quality and amount of expense in the educational state porttfolio in the Ecuador: reach of superior education and intercultural education

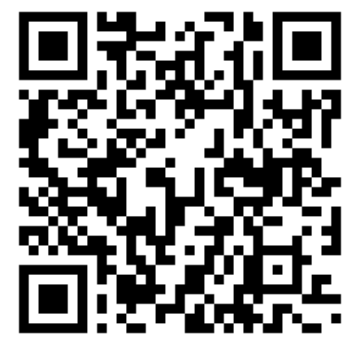

Alegría Crespo Cordovez ${ }^{* *}$

\section{Resumen}

El presente artículo recopila un análisis profundo sobre el Estado y el gasto invertido en educación con el fin de articular su presupuesto general hacia un incremento porcentual en la cartera de educación, donde estén alineados conceptos de interculturalidad, educación ambiental, desarrollo sostenible. El desarrollo histórico de la educación en el Ecuador hace que tengamos la coyuntura actual de un sistema educativo que requiere inversión y atención. Con el fin de comprender la actualidad educativa, se requiere un panorama que aborde los fenómenos sociológicos, económicos, históricos y llegar a comprender a los distintos actores sociales y su incidencia sobre la educación. Lo que se pretende es generar conciencia y reflexión social. El sistema educativo debe estar encaminado a fomentar el pensamiento crítico, así como el desarrollo de la creatividad y la implantación de metodologías innovadoras para que se manifiesten posteriormente en el sector productivo. El momento en que se

Artículo original derivado del proyecto de investigación titulado "Universidad Nacional del Rosario". Entidad financiadora: Autores, fecha de realización entre 2019.

** Ph.D en educación. Universidad Nacional del Rosario. Quito, Ecuador. E-mail: alegriacrespog@gmail.com._ ORCID: http://orcid.org/0000-0001-5716-9585.
Sinergias educativas

Enero - junio Vol. 5 -1 - 2020

http://sinergiaseducativas.mx/index.php/revista/ elSSN: 2662-6661

revistasinergia@soyuo.mx

Recepción: 11 junio 2018

Aprobación: 19 diciembre 2019

Pag 370-383

Disponible en

http://www.redalyc.org/articulo.oa? $\mathrm{id}=57356143200$ 28

Atribución/Reconocimiento-NoComercialCompartirlgual 4.0 Licencia Pública Internacional CC BY-NC-SA 4.0

https://creativecommons.org/licenses/by-ncsa/4.0/legalcode.es 
articule adecuadamente el eslabón entre la formación educativa formal en miras a un cambio social y un mejoramiento productivo nacional, se habrá llegado a una sinergia. Nos encontramos en un momento histórico en el cual la educación es gestora de un cambio necesario. En el presente artículo se analizará justamente cómo los fenómenos sociales, históricos y antropológicos se ven reflejados en el estado actual de la educación del país.

Palabras clave: formación, Posmodernidad, historia.

\section{Abstract}

This article compiles an in-depth analysis of the State and the expenditure invested in education in order to articulate its overall budget towards a percentage increase in the education portfolio, where concepts of interculturality are aligned, environmental education, sustainable development. The historical development of education in Ecuador means that we have the current juncture of an education system that requires investment and attention. In order to understand the current educational events, a picture that addresses sociological, economic, historical phenomena and come to the understanding of the different social actors and their impact on education Summary: This article compiles an in-depth analysis of the State and the expenditure invested in education in order to articulate its overall budget towards a percentage increase in the education portfolio, where concepts of interculturality are aligned, environmental education, sustainable development. The historical development of education in Ecuador means that we have the current juncture of an education system that requires investment and attention. In order to understand the current educational events, a picture that addresses sociological, economic, historical phenomena and come to the understanding of the different social actors and their impact on education is required. The aim is to raise awareness and social reflection. The educational system should be focused at promoting critical thinking, as well as the development of creativity and the implementation of innovative methodologies to be manifested later in the productive sector. The moment when the link between formal educational training in view of social change and national productive improvement is properly articulated, synergy 
will have been reached. We are at a historic moment in which education is the manager of a necessary change. This article will fairly analyze how social, historical and anthropological phenomena are reflected in the current state of education in the country.

Key words: training teacher, Posmodernity, History.

\section{Introducción}

La educación en el Ecuador y en Latinoamérica ha atravesado momentos históricos que hacen que la misma se vea influenciada por ciertos acontecimientos e ideologías marcadas por transiciones sociales y económicas. En el presente artículo se analizará justamente cómo los fenómenos sociales, históricos y antropológicos se ven reflejados en el presente educativo del país. Tomando en cuenta aportes valiosos de pensadores que marcan el camino educativo como base de una sociedad en desarrollo, el siguiente es un análisis de la educación y la importancia de la multiculturalidad en la misma.

La posmodernidad marcó una pauta para el restablecer paradigmas en un nuevo "proyecto civilizatorio" que condenó la modernidad y el mundo contemporáneo. El desasosiego de las guerras mundiales despertó un deseo de refundar la política de Estado y las relaciones internacionales en esta nueva etapa de la globalización. La educación no estuvo exenta a este replanteamiento que tenía ciertos tintes eurocéntricos.

"Sólo cuando se niega el mito civilizatorio y de la inocencia de la violencia moderna, se reconoce la injusticia de la praxis sacrificial fuera de Europa (y aún en Europa misma), y entonces se puede igualmente superar la limitación esencial de la "razón emancipadora". Se supera la razón emancipadora como "razón liberadora" cuando se descubre el "eurocentrismo" de la razón ilustrada, cuando se define la "falacia desarrollista" del proceso de modernización hegemónico" (Dussel, 2002).

Desde la Constitución del Ecuador 1998 y 2008 se tipifican nuevas dimensiones de la propuesta posmoderna que abre nuevos espacios a los pueblos ancestrales del Ecuador. Eso ha determinado que ahora 
las constituciones de por lo menos once países (Argentina, Bolivia, Brasil, Colombia, Ecuador, Guatemala, México, Nicaragua, Paraguay, Perú y Venezuela) reconozcan y acepten su multiculturalidad y que algunos, como en el caso ecuatoriano, dejen incluso traslucir el carácter «multinacional» del país (López y Küper, 1999).

Tanto en el Ecuador como en otros países de América Latina y del mundo, existe una nueva coyuntura política en la cual la multiculturalidad está ganando espacio y legitimidad.

"Esta nueva coyuntura incluye el reconocimiento por parte de los Estados de la diversidad étnica y cultural y, por ende, la necesidad de otorgar algunos derechos específicos al respecto, lo que algunos autores llaman el nuevo "constitucionalismo multicultural" (Van Cott, 2000) y su relación con la ciudadanía "étnica" o "cultural" ( Pérez-Bustillo 2001; Walsh, 2002).

Según Van Cott (2000) este modelo implica el reconocimiento de tres tipos de derechos colectivos: derechos al autogobierno, derechos especiales de representación y derechos étnicos. Pérez-Bustillo (2001) señala la omisión del reconocimiento de derechos a la autonomía territorial, elemento clave de

las demandas indígenas dirigida al reconocimiento de la "plurinacionalidad" (Walsh, 2002).

La plurinacionalidad, multietnicidad e interculturalidad vuelven a dar voz y protagonismo político a los que durante tantos siglos se intentó invisibilidad por haber sido considerados "bárbaros". La filosofía de la liberación cuestiona de lleno la problemática del ser y lo atribuible que era imputar a los pueblos amerindios una suerte de estatus "salvaje", "bárbaro", "sin alma", se justificó por medio de estas dimensiones; las prácticas esclavistas, explotadoras, expolio y "educación" para que los que eran "incivilizados" puedan "educarse". Los hitos y los aportes latinoamericanistas a esta liberación paradigmática marcaron la hoja de ruta hacia el empoderamiento de los saberes, conocimientos y educación indígenas. En Ecuador, la creación de las Universidades IKIAM e Intercultural, son fiel muestra de la materialización de 
reivindicaciones indígenas que venían fraguándose con los aportes posmodernos de figuras intelectuales de la dimensión de Dussel. En el Ecuador la legislación en favor de la interculturalidad ha alcanzado espacios educativos de suma importancia en el sistema jurídico nacional. De esta manera lo contempla el título IV, capítulo I, artículos 77, 78 de la Ley Orgánica de Educación Intercultural, donde se establece y se reconoce el Sistema Intercultural Bilingüe (SEIB), mientras que en el capítulo II del título IV de la nueva ley anteriormente mencionada, establece los fundamentos, objetivos y fines del SEIB, la legislación vigente es ambiciosa y busca ir más allá para que los alcances de interculturalidad formen parte de los nuevos contenidos, alcances transversales y las concepciones de la educación que viene desde los pueblos ancestrales y las nacionalidades indígenas en el Ecuador. Los nuevos contenidos de educación en medicina alternativa ancestral ya ocupan un espacio en la malla curricular de universidades e instituciones públicas y privadas que ya no ven al Yachag o curandero como una suerte de ser mítico que el positivismo francés del siglo XVIII había intentado enterrar.

Contrario a lo acontecido en décadas anteriores, la educación intercultural cobra fuerza trascendental inclusive en la agenda cultural, educativa y hasta en la planificación y el Plan Nacional Del Buen Vivir ecuatoriano 2013-2017, específicamente el objetivo V, que menciona el construir espacios de encuentro común y fortalecer la identidad nacional, las identidades diversas, la plurinacionalidad y la interculturalidad. El neoliberalismo y su arremetida en la práctica de políticas públicas en el estado-nación latinoamericano en la década de los noventas redujo y recortó considerablemente el presupuesto general del Estado en carteras relacionadas a inversión social y sus sub-sectores desagregados como educación, salud y servicios básicos, todo esto justificando la nueva visión de "modernización y ajuste" del Estado con la finalidad de volverlo más eficiente y reducir el aparataje estatal para reducir el déficit fiscal. Finalmente, el recorte en la inversión social significó el incremento presupuestario en el aumento de cuantía y presupuesto en el pago de las obligaciones de deuda externa, sacrificando un pilar de desarrollo como es el humano, impactando de lleno en las carteras de Estado que manejaron el rubro educación en casi todos los países 
latinoamericanos, bajo la justificación de superar la década del falso crecimiento de los setentas y la década perdida de los ochentas, con la finalidad de recortar un aparataje burocrático excesivamente grande e ineficiente, resultó mucho más grave la cura que la enfermedad.

"Suponía que no todos en las instituciones financieras internacionales o en los estados que las sostienen, estarían comprometidos con el objetivo de eliminar la pobreza, pero pensé que habría un debate abierto sobre las estrategias, que en tantas áreas parecían estar fracasando y especialmente en lo que a los pobres atañe" (Stiglitz, 2002).

El neoliberalismo en Latinoamérica socavó severamente la capacidad del Estado como eje protagónico en la elaboración, monitoreo y ejecución de política pública educativa. Lo que sucedía en Latinoamérica entre los años ochenta y noventa, a hoy de la manera más paradójica se reproduce en los centros europeos de desarrollo industrial que alguna vez fueron ícono de conquistas sociales que pudieron consolidar el "Estado de Bienestar" también conocidos como economías sociales de mercado, ahora es Europa la que recorta importantes montos fiscales que iban destinados a la educación. Las aspiraciones de un acceso universal y gratuito a una educación pública de calidad cada vez se alejan más de su propia esencia de ser. Si el dualismo descalificador de categorizar al mundo moderno desde una perspectiva de "lo bueno" es decir lo blanco, lo civilizado, lo correcto, lo acertado, el progreso y todas sus dimensiones políticas económicas y sociales, también se encargó de descalificar de lo "malo" o "otros saberes" "otras cosmovisiones" “otra educación", la teoría de la CEPAL, o teoría de la dependencia ya no sólo impacta de lleno en las zonas periféricas sino en el mismo núcleo de los polos industriales donde se va desmantelando de a poco la Educación Pública de manera cualitativa y cuantitativa.

"Esta revolución conservadora es algo nuevo, apela al progreso, la razón, la ciencia -por ejemplo, la economía- para justificar la restauración e intenta así desplazar al pensamiento y la acción progresista hacia el arcaísmo. Convierte en normas de todas las prácticas, y por lo tanto en reglas ideales, las regularidades reales del 
mundo económico abandonado a su propia lógica, la llamada ley del mercado, es decir, la ley del más fuerte" (Bourdieu, 2002).

Las políticas públicas de educación fueron perdiendo un espacio cuantitativo a la hora de ser asignados recursos para la cartera de Estado que representaba esta área pero el camino por alcanzar, quizá tiene alcances cualitativos y la búsqueda de una re conceptualización de la educación hacia donde debe ir orientada, es justamente otro desafío a la hora de aplicar políticas públicas educativas. La educación de las masas es el problema fundamental de los países en desarrollo, una educación que, liberada de todos los rasgos alienantes, constituya una fuerza posibilitadora del cambio y sea impulso de libertad. Sólo en la educación puede nacer la verdadera sociedad humana y ningún individuo vive al margen de ella. Por consiguiente, la opción se da entre una educación para la domesticación alienada y una educación para la libertad (Freire, 1978).

Paulo Freire (1978) considera a la educación y la calidad de la misma como el verdadero desafío para la transformación de los países en vías de desarrollo, teniendo ésta un alcance transversal como eje de trasformación de la realidad social de un Estado, dura tarea la que debe asumir el Ministerio de Educación en el Ecuador a la hora de articular saberes ancestrales, concepciones y cosmovisiones multiétnicas y verdaderos contenidos en malla académica a la hora de trasformar la calidad educativa en un país que hace esfuerzos importantes para reconocer "paradigmas educativos paralelos". La Universidad Intercultural de las Nacionalidades y Pueblos Indígenas «Amawtay Wasi» (Casa de la Sabiduría), es una propuesta de educación superior desde el movimiento indígena y tiene como patrocinadores a la Confederación de Nacionalidades Indígenas del Ecuador (CONAIE) y al Instituto Científico de Culturas Indígenas (ICCI), Amawta Runakunapak Yachay (ARY). Es el resultado de un largo proceso de trabajo, debate y sistematización donde participó un equipo interdisciplinario conformado por directivos, docentes, comuneros, investigadores y profesionales indígenas y no indígenas (Sarango, 2004). Esto refleja la necesidad de integrar la multiculturalidad en la educación que nos identifica, que nos caracteriza. 
Las relaciones entre el primero, el segundo y el tercer sector (estado, sector privado y ONGs) deben ser interdependientes cuando se genera política pública educativa para poder articular a todos los actores. No puede ser excluyente ni discriminatoria pero debe quedar claro que la mercantilización de la educación superior y privada podría ser una amenaza que lleve a la alienación a quienes opten por carreras de tercero y cuarto nivel, el Estado debe asumir su papel histórico que le permita establecer políticas públicas que sirvan de herramienta para identificar si existe una sobreoferta de carreras en un mercado saturado como podría resultar con carreras como Derecho o Medicina, a su vez la calidad educativa que impartan las universidades públicas y privadas, deben buscar un incremento cualitativo y representativo en carreras de ciencias denominadas "blandas" y "duras", no se puede concebir un proceso de consolidación en la calidad de la educación en un escenario que excluya al estado. La nueva Ley Orgánica de Educación Superior y sus entes reguladores CES (Consejo de Educación Superior), CEAACES (Consejo de Evaluación, Acreditación y Aseguramiento de la Calidad de la Educación Superior) buscan en principio romper de alguna manera la lógica mercantil en la que estaba cayendo la institución universitaria y la oferta de pregrados en el Ecuador donde se llegó a criticar por parte del Presidente de la República, Rafael Correa Delgado, la existencia de "universidades de garaje", es decir, instituciones que no tenía infraestructura física, académica o un cuerpo de docentes con la suficiente preparación académica para brindar servicios de educación superior con niveles óptimos de calidad.

Estas políticas de Estado buscan volver a las bases académicas y no mercantilistas en principio, buscan corregir los errores que ciertas universidades estarían incurriendo al ofertar sin discriminación carreras universitarias, así como la promoción de carreras poco funcionales y la excesiva apuesta de carreras en ciencias blandas, lo cual no responderían a una lógica que permita entrar en procesos de Industrialización Sustitutiva de Importaciones a la que el Estado busca en su Plan Nacional del Buen Vivir (2013-2017) en la búsqueda de dirigir las políticas públicas de educación hacia un engranaje que genere un recambio de la matriz productiva romper con el modelo de desarrollo Agro Exportador que por el contrario 
profundizó la inequidad tanto en renta como en riqueza nacional. La Amazonía y sus pueblos ancestrales no sólo que tuvieron desatención estatal en educación, servicios básicos e infraestructura sino que los pasivos ambientales que dejó la actividad petrolera necesitó de un proyecto piloto que trabaje en conjunto con las comunidades y nacionalidades indígenas, este fue el caso del Plan Remediación Ambiental y Social, PRAS, impulsado en el actual gobierno desde el Ministerio del Ambiente para en paralelo, articular ejes transversales del Plan Nacional del Buen Vivir como el Objetivo 5 anteriormente mencionado.

El modelo Agro-Exportador generó tendencias sociales de comportamiento y la educación respondió a esta lógica mientras se hipotecaba el capital natural y la biodiversidad en el Ecuador. Así, la mercancía había alcanzado la ocupación total de la vida social. La relación con la mercancía no sólo era visible, sino que era lo único visible: el mundo que se ve es su mundo.

"La producción económica moderna extiende su dictadura extensiva e intensivamente. Su reinado ya está presente a través de algunas mercancías-vedettes en los lugares menos industrializados, en tanto que dominación imperialista de las zonas que encabezan el desarrollo de la productividad. En estas zonas avanzadas el espacio social es invadido por una superposición continua de capas geológicas de mercancías. En este punto de la "segunda revolución industrial" el consumo alienado se convierte para las masas en un deber añadido a la producción alienada. Todo el trabajo vendido de una sociedad se transforma globalmente en mercancía total cuyo ciclo debe proseguirse. Para ello es necesario que esta mercancía total retorne fragmentariamente al individuo fragmentado, absolutamente separado de las fuerzas productivas que operan como un conjunto. Es aquí por consiguiente donde la ciencia especializada de la dominación debe especializarse a su vez: se fragmenta en sociología, psicotecnia, cibernética, semiología, etc., vigilando la autorregulación de todos los niveles del proceso" (Debord, 2002).

La producción económica que obedece al monocultivo extensivo, el modelo agro exportador y la lógica de exclusión en la que jugó el capital cultural en el Ecuador de los siglos XIX y XX fue el resultado de una lógica engranada a la lógica del capital y las élites, es decir 
que la educación también jugó un papel de exclusión, descalificación o categorización de ciudadanos que podían insertarse en el aparato productivo o por el contrario responderían a la masa marginal que a la postre significaría una abundante mano de obra barata, según la lógica marxista. "La institución escolar impone a menudo con una brutalidad psicológica muy grande sus juicios tajantes y sus veredictos inapelables que colocan a todos los alumnos en una jerarquía única de formas de excelencia" (Bourdieu, 1997).

Foucault (1983) va un paso más allá y critica inclusive el supuesto éxito de las sociedades desarrolladas en sus sistemas educativos y su lógica moderna y positivista de segregación perpetuación de modelos de poder con visión reduccionista donde las élites responden a una relación tripartita de familias, capital cultural y capital. Foucault critica esta lógica y considera que la visión positivista, reduccionista, rompiendo la lógica de esta educación que respondería a simples reducciones dogmáticas de verdades absolutas, reduciendo a la educación a simples estadísticas.

"En Japón como en Francia, los padres abrumados, los jóvenes aburridos y los empleadores decepcionados por los productos de una enseñanza que encuentran inadaptada, son las víctimas impotentes de un mecanismo que no es otra cosa que el efecto acumulado de sus estrategias acarreadas por la lógica de la competencia o de la lucha de todos contra todos" (Bourdieu, 1997).

De esta manera la nueva política pública del Ecuador deberá mirar con mucha prudencia, qué elementos podría adoptar del exterior en su nueva redefinición de paradigmas educativos y qué deberá ser desechado.

La escuela, el colegio, los hospitales y las academias de guerra según Foucault y Deleuze vienen a convertirse de algún modo en espacios de concentración y control que responderán según Foucault a una consolidación de posturas de poder y control en ámbitos de formación y disciplina donde la educación sería la herramienta para instrumentalizar esta lógica que violentaría el ejercicio de la disciplina del saber para imponer el ejercicio de la disciplina de la imposición. 
"Todos los centros de encierro atraviesan una crisis generalizada: La familia es un interior en crisis, como lo son los demás interiores (el escolar, el profesional, etc.) Los ministros competentes anuncian constantemente las supuestamente necesarias reformas. Reformar la escuela, reformar la industria, el hospital, el ejército, la cárcel; pero todos saben que a un plazo más o menos largo, esas instituciones están ya acabadas. Solamente se pretende gestionar su agonía y mantener a la gente ocupada mientras se instalan esas nuevas fuerzas que ya está llamando a nuestras puertas. Se trata de las sociedades de control que están sustituyendo a las disciplinarias" (Deleuze, 1996).

La política pública ecuatoriana enfocada hacia la educación necesitará consolidarse en un talento humano que tenga la suficiente claridad académica y política que le permita seleccionar, identificar y consolidar política pública con un sistema de alertas tempranas que responda a las máximas que exige la Constitución y la nueva Ley Orgánica de Educación Superior. En este ejercicio de consolidación de políticas, los servidores públicos deberán tener capacitación suficiente que les permita comprender, cuales elementos de la modernidad y el positivismo deberán mantenerse y cuales elementos del pos estructuralismo y la posmodernidad deban marcar la pauta de lo que se debe abolir para que el Ecuador pueda alinear su política pública educativa, a su Plan Nacional de Desarrollo y sus objetivos, con el fin de que la ejecución de su agenda y la instrumentalización de la misma de SENPLADES sea funcional y pragmática.

La nueva matriz productiva, el nuevo modelo de desarrollo no son más que una reorientación en las políticas públicas de la educación y todas sus cosmovisiones para que la profesionalización de carreras de grado y posgrado, obedezcan a un orden de reconfigurar el modelo de desarrollo nacional que ya no busque una lógica epidérmica de "aparente progreso" que no signifique verdadero desarrollo para el país, que permita reducir vulnerabilidad y dependencia en su relación con otros estados. La educación y sus políticas públicas son eje trasversal para romper con esas prácticas caducas que apostaron a una visión mercantilista, moderna, positivista y hasta neoliberal que se ha podido romper en el establecimiento de la nueva política pública educativa. 
El desarrollo de un Estado no deberá entonces sacrificar su talento humano y deberá socializar las ganancias y los logros, disminuyendo en la mayor medida el costo social que podría significar el "progreso" y el poder salir del subdesarrollo. Los "Tigres Asiáticos" y los países desarrollados podrán ser un referente hasta cierto punto, ya que la nueva política pública deberá entender que la educación será un puntal en el proceso de reconversión de paradigmas educativos $\mathrm{y}$ formativos de talento humano que permitan una reactivación del aparato productivo, la disminución de la inequidad en la renta y la riqueza y el apuntalamiento del único camino que se podrá mantener a largo plazo como es el desarrollo sostenible y su principal herramienta, la educación ambiental.

El Ecuador emerge ante una dirección de satisfacer la matriz productiva con el fin de generar sus propios productos. La educación es la base para formar una sociedad preparada que se forme para los nuevos retos y desafíos que requieren los entes productivos. Desde la educación inicial, el currículo debe ser replanteado en base al Constructivismo, pero sobretodo a destrezas creativas e innovadoras. Esto necesita un cambio de paradigmas del sistema educativo para permitir que el estudiante sea el centro del proceso de aprendizaje y gestor protagonista de sus conocimientos y por ende, del servicio como ciudadano a su realidad, a su entorno y a la vez se reconfigure nuevos paradigmas pedagógicos que no tengan como prioridad la coacción. Las cárceles, los hospitales y las escuelas presentan similitudes porque sirven para la intención primera de la civilización: la coacción. (Foucault, 1983). A lo largo del período educativo, todos los actores del mismo deben tener la misma visión: generar conciencia social e individuos críticos, creativos y proactivos. Nos encontramos en un momento histórico en el cual la educación es gestora de un cambio necesario.

\section{Conclusiones}

Como un esbozo general, el abordaje crítico de los paradigmas caducos de educación de corte positivista/moderno deben ser abordados por una nueva dimensión constructivista-posmoderna, inclusiva que entienda la importancia de cubrir "otros saberes" y "otros conocimientos" que rompan de una vez con la concepción del "Pensamiento único - eurocéntrico". Este papel debe abordar el 
Estado y articular su presupuesto general hacia un incremento porcentual en la cartera de educación, donde estén alineados conceptos de interculturalidad, educación ambiental, desarrollo sostenible - agenda XXI, Constitución, Políticas de Estado, SENPLADES y el Plan Nacional del Buen Vivir, para que apostando a la educación y sus nuevos paradigmas, se abone el terreno ideal en talento humano que a la postre será la base sustancial y generacional del salto cuántico del subdesarrollo hacia el desarrollo. La conjugación de estos factores más la participación activa del estado, sector privado y organizaciones sin fines de lucro, podrán reactivar el aparato productivo en todas las áreas que el estado requiera. El desafío está ahí, los referentes de educación tienen precedentes como Singapur o Corea del Sur. El reto está en superar la concepción quizá reduccionista del desarrollo económico que busca crecimiento y equidad pero podría olvidar la sostenibilidad y las externalidades ambientales y una educación integral, participativa, incluyente e Intercultural.

\section{Referencias}

Bourdieu, P. (2002). Pensamiento y acción/Thought and action. Buenos Aires: Libros del Zorzal

Bourdieu, P. (1997). Capital cultural, escuela y espacio social. Buenos Aires: Siglo XXI

Debord, G. (1967). La sociedad del espectáculo. Paris: Champ Libre.

Deleuze, G. (1995). Conversaciones. Valencia: Pre-textos.

Dussel, E. (2000). Europa, modernidad y eurocentrismo.Argentina: Clacso.

Foucault, M. (1983). Vigilar y castigar: nacimiento de la prisión. Buenos Aires: Siglo XXI

Freire, P. (1978). La educación como práctica de la libertad. Buenos Aires: Siglo XXI. 
LOEI. (2011). Ley Orgánica de Educación Intercultural - Registro Oficial No. 417. Recuperado de https://educacion.gob.ec/wpcontent/uploads/downloads/2017/05/Ley-Organica-EducacionIntercultural-Codificado.pdf

López, L. E., \& Küper, W. (1999). La educación intercultural bilingüe en América Latina: balance y perspectivas. Revista Iberoamericana de Educación, 20(1), 17-85.

Pérez-Bustillo, C. (2001). "De la ciudadanía multicultural a la interculturalidad: intentos recientes de reconstrucción del estado en México, Guatemala y Colombia," Ponencia presentada en LASA, Washington D.C.

SENPLADES. (2014). Plan Nacional de Desarrollo - Plan Buen Vivir. Recuperado de http://observatorioplanificacion.cepal.org/es/planes/plan-nacionalde-desarrollo-todos-por-un-nuevo-pais-de-colombia-2014-2018

Sarango, L. (2004). Universidad Intercultural de las Nacionalidades y Pueblos Indígenas «Amawtay Wasi». Ecuador/Chinchaysuyu. Ecuador: FLACSO Andes

Stiglitz, J. E. (2002). El malestar en la globalización. Bogotá: Taurus

Van Cott, Donna Lee (2000). The friendly liquidation of the past: The politics of diversity in Latin America. Pittsburgh: University of Pittsburgh Press.

Walsh, C. (2002). Interculturalidad, reformas constitucionales y pluralismo jurídico. Judith Salgado (comp.). Justicia indigena. Aportes para un debate, 23-36. 